\title{
Architectural Approach to eHealth for Enabling Paradigm Changes in Health
}

\author{
B. Blobel ${ }^{1}$ \\ ${ }^{1}$ eHealth Competence Center, University Hospital Regensburg, Regensburg, Germany
}

\begin{abstract}
Summary
Objectives

For improving safety and quality of care as well as efficiency of health delivery under the well-known burdens, health services become specialized, distributed, and therefore collaborative, thereby changing the health service paradigm from organization-centered over processcontrolled to personal health (pHealth).
\end{abstract}

\section{Methods}

Personalized eHealth services provided independent of time and location have to be based on advanced technical paradigms of mobile, pervasive and autonomous computing, enabling ubiquitous health services. Personalized eHealth systems require a multidisciplinary approach including medicine, informatics, biomedical engineering, bioinformatics and the omics disciplines but also legal and regulatory affairs, administration, security, privacy and ethics, etc. Interoperability between different components of the intended system must be provided through an architecture-centric, model-driven, formalized process.

\section{Results}

In order to analyze, design, specify, implement and maintain such an interactive environment impacted by so many different domains, a formal and unified methodology for system analysis and design has been developed and deployed, based on an overall architectural framework. The paper introduces the underlying paradigms, requirements, architectural reference models, modeling and formalization principles as well as development processes for comprehensive serviceoriented personalized eHealth interoperability chains, thereby exploiting all interoperability levels up to service interoperability. A special focus is put on ontologies and knowledge representation in the context of eHealth and pHealth solutions. Furthermore, EHR solutions, security requirements, existing and emerging standards, and educational challenges for realizing personalized pHealth are briefly discussed.

\section{Conclusion}

For personal health, bridging between disciplines including ontology coordination is the crucial demand. All aspects of the design and development process have to be considered from an architectural viewpoint.

\section{Keywords}

Ontology, Knowledge Representation, semantic interoperability, Personal health, system architecture, universal logic, ubiquitous care

\section{DOI}

10.3414/ME9308

The full English version can be found in /Methods of Information in Medicine 2010 49 2: $123-134 /$ or http://www.schattauer.de/de/magazine/ue bersicht/zeitschriften-az/methods/issue/special/manuscript/1261 7/show.html. 